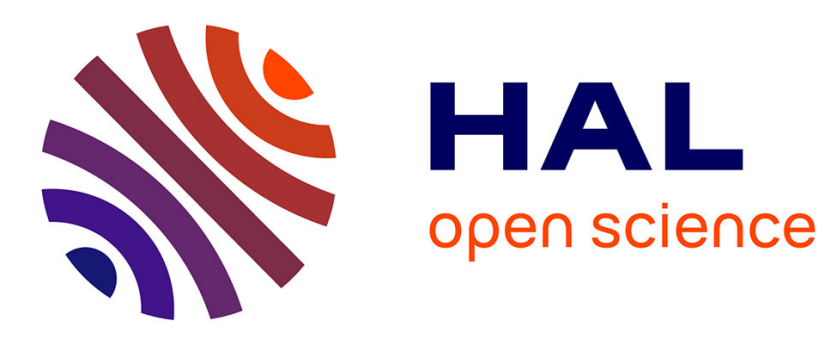

\title{
On the symmetry of FIR filter with linear phase
}

Stéphane Paquelet, Vincent Savaux

\section{To cite this version:}

Stéphane Paquelet, Vincent Savaux. On the symmetry of FIR filter with linear phase. Digital Signal Processing, 2018, 81 (2018), pp.57 - 60. 10.1016/j.dsp.2018.07.011 . hal-01849361

\section{HAL Id: hal-01849361 https://hal.science/hal-01849361}

Submitted on 31 Jul 2018

HAL is a multi-disciplinary open access archive for the deposit and dissemination of scientific research documents, whether they are published or not. The documents may come from teaching and research institutions in France or abroad, or from public or private research centers.
L'archive ouverte pluridisciplinaire HAL, est destinée au dépôt et à la diffusion de documents scientifiques de niveau recherche, publiés ou non, émanant des établissements d'enseignement et de recherche français ou étrangers, des laboratoires publics ou privés. 
DOI : https://doi.org/10.1016/j.dsp.2018.07.011

(C) 2018. This manuscript version is made available under the CC-BYNC-ND 4.0 license 


\title{
On the Symmetry of FIR Filter with Linear
}

\section{Phase}

\author{
Stéphane Paquelet, Vincent Savaux*
}

\begin{abstract}
This paper deals with signal processing theory related to finite impulse response (FIR) filters with linear phase. The aim is to show a new and complete proof of the equivalence between linear phase and symmetry or antisymmetry of the real coefficients of the filter. Despite numerous proofs are available in the literature, they are usually incomplete, even though the result is commonly used by the signal processing community. We hereby address a pending issue in digital signal processing: we first prove the uniqueness of the group delay for any decomposition amplitude-phase of the frequency response. Based on this first step, we then derive a complete proof of the equivalence: a FIR filter has (anti)symmetric coefficients if and only if the phase is linear. It must be emphasized that this brief paper deals with theoretical aspects of FIR filters.
\end{abstract}

Keywords- FIR Filter, Group Delay, Linear Phase.

\section{INTRODUCTION}

Analysis and design of digital finite impulse response (FIR) filters with linear phase are basics in the field of signal processing [1]-[4]. Such filters are widely used in electronic devices for their inherent stability. Furthermore, numerous techniques for FIR filter design and applications have been proposed, from early 70's [2] to the present day [5], [6]. However, unlike papers like [2], [5], [6] which deal with design and applications of FIR filters, the present paper is dedicated to theoretical aspects related to FIR filters.

\footnotetext{
Manuscript submitted in June 2017

Stéphane Paquelet and Vincent Savaux are with IRT $\mathrm{b}<>$ com, Rennes, FR

* Corresponding author: e-mail: vincent.savaux@b-com.com Phone: +33 256358216.
} 
It is commonly accepted that a FIR filter has a linear phase if and only if the taps are either symmetric or antisymmetric. However, to the best of our knowledge, although this result is commonly accepted, the proof leading to this fundamental property is not satisfactorily demonstrated in the literature [1], [7], [8], in which mathematical shortcuts are used. In particular, showing that a (anti)symmetric filter has a linear phase is straightforward (see [7] for instance), whereas the inverse implication requires more difficult developments. Otherwise, the authors of [8] made an attempt to prove the double implication, but they inherently presupposed some features regarding the group delay and the differentiability of the frequency response of FIR filters.

In this paper, we revisit the proof of the equivalence between linear phase and (anti)symmetry of FIR filters. To this end, we first prove the existence of the decomposition module/phase of the frequency response of the filter, where both the module and phase functions are at least of class $\mathcal{C}^{1}$. Furthermore, we show that the group delay is unique for any decomposition. To the best of our knowledge, both proofs are original contributions of this paper, and they are a mandatory prerequisite for the proof of the equivalence between linear phase and (anti)symmetry, since it justifies the existence of both the phase/module decomposition and the group delay.

Once these properties are justified, we can prove the equivalence between linear phase and (anti)symmetry of FIR filters with proper assumptions. Showing that a FIR filter has a linear phase if it is (anti)symmetric is not tackled in this paper, since it has been undertaken in [1], [7]. However, we focus on the inverse implication, i.e. we prove that a FIR filter is (anti)symmetric if the phase is linear. This is another original contribution of this paper. Moreover, we consider a very general approach in the sense that the filter coefficients are considered as elements of a mathematical sequence, and no assumption about FIR filters features is made. From this, the proof is based on the analysis of the linear independence of sin functions. To summarize, this paper closes the theoretical concepts related to linearity of the phase of FIR filters, (anti)symmetry of the taps, and uniqueness of the group delay.

The paper is organized as follows: in Section II, we prove the existence of a decomposition module/phase of the frequency response of any FIR filter, and the uniqueness of the group delay. In Section III, we show the following implication: if a FIR filter has a linear phase, then the coefficients are symmetric or antisymmetric, which completes the existing results concenring the 
inverse implication. Finally, Section IV concludes this paper.

\section{BaCKGround AND Prerequisites}

\section{A. Standard Mathematical Notations}

The following notations are used throughout the paper: the class $\mathcal{C}^{k}(\mathbb{A})$ of a function $f$ means that the $k$-th derivative of $f$ exists and is continuous on the set $\mathbb{A}$; the braces $\{$.$\} define discrete$ sets, and $|f|$ is the modulus of $f$. The mathematical equivalence between two assertions $(\mathcal{A}) \Leftrightarrow(\mathcal{B})$ means that we have the double implication $(\mathcal{A}) \Rightarrow(\mathcal{B})$ and $(\mathcal{A}) \Leftarrow(\mathcal{B})$.

\section{B. Model}

We consider a finite impulse response (FIR) filter composed of $M$ real coefficients $\left\{h_{m}, 0 \leq\right.$ $m \leq M-1\}$. The filter frequency response is obtained by means of the discrete-time Fourier transform (DTFT) as follows:

$$
H(\nu)=\sum_{m=0}^{M-1} h_{m} e^{-2 j \pi m \nu}
$$

where $\nu \in I=\left[-\frac{1}{2}, \frac{1}{2}\right]$ is the normalized frequency. In order to highlight the amplitude response of the FIR (not to be confused with the magnitude $|H|[2]$, [4]), it is more relevant to consider, if it exists, the following decomposition of $H(\nu)$ :

$$
H(\nu)=H_{r}(\nu) e^{j \phi(\nu)}
$$

where $H_{r}$ and $\phi$ must be (at least) $\mathcal{C}^{1}(\mathbb{R})$ functions. Note that the differentiability class of $H_{r}$ is rarely discussed in the literature, even it is usually stated that it is sufficient for $H_{r}$ to be $\mathcal{C}^{0}(\mathbb{R})$. Now, it must be emphasized that the existence of the group delay, defined as $\alpha=-\frac{1}{2 \pi} \frac{d \phi(\nu)}{d \nu}$, presupposes the existence of a decomposition $(2)$ with $\phi$ a $\mathcal{C}^{1}(\mathbb{R})$ function, such that the derivative of $\phi$ is unique for any possible decomposition. As a consequence, we deduce that $H_{r}$ is necessarily $\mathcal{C}^{1}(\mathbb{R})$ since $H_{r}(\nu)=H(\nu) e^{-j \phi(\nu)}$, where $H(\nu)$ is $\mathcal{C}^{\infty}$ by construction (1). Such assertions are proved hereafter. 


\section{Existence of the Decomposition (2)}

Theorem 1. $\forall \nu \in I=\left[-\frac{1}{2}, \frac{1}{2}\right], \exists H_{r}$ and $\phi$ of $\operatorname{class} \mathcal{C}^{1}(\mathbb{R})$, such that $H(\nu)$ defined in (1) can be decomposed as $H(\nu)=H_{r}(\nu) e^{j \phi(\nu)}$.

Proof. Since any polynomial can be decomposed as a product of monomials, one can show that every monomial is $\mathcal{C}^{1}(\mathbb{R})$ to prove that the polynomial is $\mathcal{C}^{1}(\mathbb{R})$. For the sake of clarity, we rewrite (1) by using the $z$ transform notation, where $z=e^{2 j \pi \nu}$. Thus, if we suppose that $h_{M-1} \neq 0,(1)$ is factorized as:

$$
H(z)=\sum_{m=0}^{M-1} h_{m} z^{-m}=h_{M-1} \prod_{m=0}^{M-1}\left(z^{-1}-z_{m}^{-1}\right),
$$

where $z_{m}^{-1}$ is the root of the monomial $\left(z^{-1}-z_{m}^{-1}\right)$. Let us denote $z_{m}=\rho_{m} e^{j \theta_{m}}$, where $\left(\rho_{m}, \theta_{m}\right) \in$ $\mathbb{R}^{2}$. We aim to show that, for any $m \in\{0,1, . ., M-1\}$, the monomials can be expressed as

$$
z^{-1}-z_{m}^{-1}=H_{m}(\nu)=H_{m, r}(\nu) e^{j \phi_{m}(\nu)}
$$

where $H_{m, r}$ and $\phi_{m}$ are $\mathcal{C}^{1}(\mathbb{R})$ functions, which will be proved afterward.

Two cases have to be dealt with, according to $\rho_{m}=1$ or $\rho_{m} \neq 1$ :

- if $\rho_{m}=1$, some straightforward trigonometric manipulations allow us to rewrite the monomial as follows:

$$
\begin{aligned}
z^{-1}-z_{m}^{-1} & =e^{-2 j \pi \nu}-e^{-j \theta_{m}} \\
& =2 \sin \left(\pi \nu-\frac{\theta_{m}}{2}\right) e^{-j\left(\pi \nu+\frac{\theta_{m}}{2}+\frac{\pi}{2}\right)},
\end{aligned}
$$

where we identify $H_{m, r}(\nu)=2 \sin \left(\pi \nu-\frac{\theta_{m}}{2}\right)$ and $\phi_{m}(\nu)=-\left(\pi \nu+\frac{\theta_{m}}{2}+\frac{\pi}{2}\right)$. Note that both functions are of class $\mathcal{C}^{\infty}(\mathbb{R})$, hence $\mathcal{C}^{1}(\mathbb{R})$.

- If $\rho_{m} \neq 1, H_{m}$ is of class $\mathcal{C}^{\infty}$ from $\mathbb{R}$ to $\mathbb{C}^{*}$, as $H_{m}(\nu) \neq 0$ for any $\nu \in I$. Then, from lifting property of the map $p: \mathbb{R} \mapsto S^{1}$ given by $p(\nu)=\frac{H_{m}(\nu)}{\left|H_{m}(\nu)\right|}$, where $S^{1}$ is the unit circle (see [9] for more details), we know that the polar form of $H_{m}(\nu)=H_{m, r}(\nu) e^{j \phi_{m}(\nu)}$ exists, with $H_{m, r}=\left|H_{m}\right|$ and $\phi_{m}$ of the same class as $H_{m}$. Hence $H_{m, r}$ and $\phi_{m}$ are of class $\mathcal{C}^{1}(\mathbb{R})$. This concludes the proof. 
The existence of the decomposition in (2) being proved, we can now prove that the group delay is unique for any decomposition module/phase of $H$. To this end, we show the uniqueness of the derivative of $\phi$.

\section{Uniqueness of the Derivative of $\phi$}

Theorem 2. The derivative of $\phi$ is unique for any decomposition of $H$ defined in (2).

Proof. Let $\nu_{n}$ and $\nu_{n+1}$ be two consecutive zeros of $H$. We consider the interval $\left.J_{n}=\right] \nu_{n}, \nu_{n+1}[$, where $H(\nu) \neq 0$. Furthermore, we denote by $\left(H_{r, 1}, \phi_{1}\right)$ and $\left(H_{r, 2}, \phi_{2}\right)$ two possible decompositions of $H$. Thus, for any $\nu \in J_{n}$, since $\left|H_{r, 1}(\nu)\right|=\left|H_{r, 2}(\nu)\right|$, we have $H_{r, 1}(\nu)=\kappa(\nu) H_{r, 2}(\nu)$, where $\kappa(\nu)=\{ \pm 1\}$. Therefore, we deduce that:

$$
e^{j\left(\phi_{1}(\nu)-\phi_{2}(\nu)\right)}= \pm 1
$$

and then $\frac{\phi_{1}(\nu)-\phi_{2}(\nu)}{\pi}=k_{n}$, where $k_{n} \in \mathbb{Z}$. We proved that $\phi_{1}$ and $\phi_{2}$ are continuous on $\mathbb{R}$, hence

they are on $\bar{J}_{n} \ni \nu_{n+1}$, thus $\frac{\phi_{1}\left(\nu_{n+1}\right)-\phi_{2}\left(\nu_{n+1}\right)}{\pi}=k_{n}$, and on $\bar{J}_{n+1} \ni \nu_{n+1}$, thus $\frac{\phi_{1}\left(\nu_{n+1}\right)-\phi_{2}\left(\nu_{n+1}\right)}{\pi}=$ $k_{n+1}$. Then, for any $n$, we deduce that $k_{n}=k_{n+1}=k$, and $\kappa(\nu)=(-1)^{k}$. To summarize, the above results show that every possible decomposition of $\mathrm{H}$ can be deduced from a unique one $\left(H_{r}, \phi\right)$ as $\left((-1)^{k} H_{r}, \phi+k \pi\right)$. Finally, this points out that the derivative of $\phi$ (which is proportional to the group delay if the phase is linear) is unique and independent of the decomposition, which concludes the proof.

Such as aforementioned, it is usual to consider $H_{r}(\nu)=|H(\nu)|$ in the literature, or otherwise the differentiability class of $H_{r}$ and $\phi$ is not discussed [2], [4]. However, it can be noticed that if the $\mathcal{C}^{0}(\mathbb{R})$ condition is imposed to $H_{r}$ instead of $\mathcal{C}^{1}$, as in the case of the modulus on the zeros of $H$, then $\phi$ is not $\mathcal{C}^{1}(\mathbb{R})$ and the group delay is not defined anymore. Furthermore, it must be noticed that all the possible decompositions $\left((-1)^{k} H_{r}, \phi+k \pi\right), k \in \mathbb{Z}$, are of the same class. Since at least one is of class $\mathcal{C}^{\infty}$ (see proof of Theorem 1 for instance), then all decompositions are $\mathcal{C}^{\infty}$ 


\section{Symmetry of FiR Filter with Linear Phase}

The aim of this section is to show that any FIR filter has a linear phase if and only if their real coefficients are either symmetric or antisymmetric. Mathematically, this can be expressed as

$$
\begin{aligned}
(\mathcal{A})\left\{\begin{array} { l } 
{ H ( \nu ) = H _ { r } ( \nu ) e ^ { j \phi ( \nu ) } } \\
{ \phi ( \nu ) = \beta - 2 \pi \alpha \nu }
\end{array} \Leftrightarrow \left\{\begin{array}{l}
\alpha=\frac{M-1}{2} \\
\beta \in\left\{k \pi, \frac{\pi}{2}+k \pi\right\} \quad(\mathcal{B}), \\
h_{m}=\varepsilon_{\beta} h_{M-1-m}
\end{array}\right.\right. \\
\varepsilon_{\beta}=\left\{\begin{array}{ll}
1, & \text { if } \beta=\frac{\pi}{2}+k \pi \\
-1, & \text { if } \beta=k \pi
\end{array},\right. \\
k \in \mathbb{Z},(\alpha, \beta) \in \mathbb{R}^{2},
\end{aligned}
$$

The proof of the implication $(\mathcal{A}) \Leftarrow(\mathcal{B})$ is straightforward and frequently proposed in the literature such as [1], [7], therefore it is not reported in this paper. However, we propose an original proof of the implication $(\mathcal{A}) \Rightarrow(\mathcal{B})$. This is undertaken by considering a very general context, where no a priori constraint on the values of the filter coefficients $h_{m}$ is imposed. In particular, it is mathematically possible to assume $h_{0}=0$ or $h_{M-1}=0$, even though, in practice, it is unlikely that the extremal coefficients are zero.

\section{A. Preliminaries}

Let $J$ be an interval $J \subset I$ such as $\forall \nu \in J, \sum_{m=0}^{M-1} h_{m} \cos (2 \pi m \nu) \neq 0$. The existence of $J$ is guaranteed since the number of roots of a trigonometric polynomial is finite. If non-zero FIR filters with linear phase exists, for any $\nu \in J$, the decomposition of the equation $(1)=(2)$ into real and imaginary parts yields

$$
\left\{\begin{array}{l}
H_{r}(\nu) \cos (\beta-2 \pi \alpha \nu)=\sum_{m=0}^{M-1} h_{m} \cos (2 \pi m \nu) \\
H_{r}(\nu) \sin (\beta-2 \pi \alpha \nu)=-\sum_{m=0}^{M-1} h_{m} \sin (2 \pi m \nu)
\end{array}\right.
$$

Hence, for any $\nu \in J$, we can write:

$$
\tan (\beta-2 \pi \alpha \nu)=\frac{-\sum_{m=0}^{M-1} h_{m} \sin (2 \pi m \nu)}{\sum_{m=0}^{M-1} h_{m} \cos (2 \pi m \nu)} .
$$

Then, after some straightforward trigonometric manipulations, we obtain:

$$
\sum_{m=0}^{M-1} h_{m} \sin (2 \pi \underbrace{(\alpha-m)}_{\alpha_{m}} \nu+\beta)=0,
$$


where $\alpha_{m}$ is defined for the sake of readability. In the following, we analyze the linear independence of the elements of set $\left\{\sin \left(2 \pi \alpha_{m} \nu+\beta\right), 0 \leq m \leq M-1, \nu \in J\right\}$ in order to deduce the symmetry/antisymmetry properties of the coefficients $h_{m}$.

It is usually claimed in the literature that no additional result can be deduced from (13), except that "obvious" non-null solutions are obtained when $\beta \in\left\{k \pi, \frac{\pi}{2}+k \pi\right\}, k \in \mathbb{Z}$, and $\alpha=\frac{M-1}{2}$, and in that case the filter is either symmetric or antisymmetric. However, this is a shortcut that should be mathematically proved. We hereafter provide an original and complete proof of $(\mathcal{A}) \Rightarrow(\mathcal{B})$ from $(13)$, from which we deduce the $\alpha$ value based on mathematical considerations.

\section{B. Linear Independence and Notations}

It must be emphasized that if the $\left|\alpha_{m}\right|$ elements in (13) are all distinct, then the functions $\nu \in J \mapsto \sin \left(2 \pi \alpha_{m} \nu+\beta\right), 0 \leq m \leq M-1$, are linearly independent, except if one of the sin function is null. In any case, the only solution is either the null filter, or a trivial single-coefficient filter. As a consequence, we deduce that a non-trivial solution exists only if there are at least two different indexes $m$ and $m^{\prime}$ such that $\left|\alpha_{m}\right|=\left|\alpha_{m^{\prime}}\right|$, or equivalently $\alpha_{m}=-\alpha_{m^{\prime}}$, and hence:

$$
\frac{m+m^{\prime}}{2}=\alpha
$$

This remark suggests splitting the index of the sum in (13) into two subsets: one gathering of indexes $m$ for which a value $\left|\alpha_{m}\right|$ appears only once, denoted by $E_{1}$, and one for which $\left|\alpha_{m}\right|$ appears twice, denoted by $E_{2}$. By construction, $E_{1} \cup E_{2}=\{0,1, . ., M-1\}$ and $E_{1} \cap E_{2}=\{\emptyset\}$ : it is a partition of $\{0,1, . ., M-1\}$.

Accordingly, the development of the sum in (13) leads to the following expression:

$$
\begin{aligned}
& \sum_{m \in E_{1}} h_{m} \sin \left(2 \pi \alpha_{m} \nu+\beta\right) \\
& +\sum_{m, m^{\prime} \in E_{2}}\left[\left(h_{m}+h_{m^{\prime}}\right) \cos \left(2 \pi \alpha_{m} \nu\right) \sin (\beta)\right. \\
& \left.+\left(h_{m}-h_{m^{\prime}}\right) \sin \left(2 \pi \alpha_{m} \nu\right) \cos (\beta)\right]=0 .
\end{aligned}
$$

\section{C. $h$ Symmetry and $\beta$ Possible Values}

$E_{1}$ Examination: By linear independence of the functions $\sin \left(2 \pi \alpha_{m} \nu+\beta\right)$, we deduce that if $m \in E_{1}, h_{m}=0$, except in the case of a null sin function, i.e. $\alpha_{m}=0$ and $\beta=k \pi, k \in \mathbb{Z}$. 


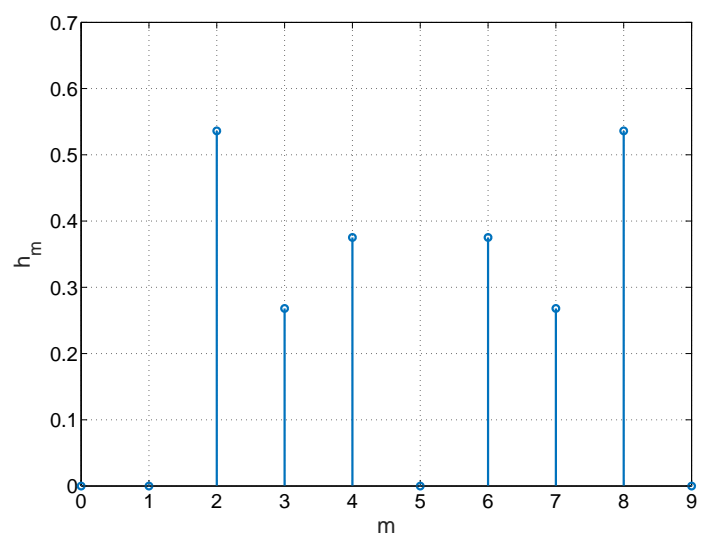

Fig. 1. Filter coefficients, $\operatorname{Min}=2$ and $\operatorname{Max}=8$.

In the latter case, $m=\alpha$ and $h_{\alpha} \in \mathbb{R}$. Moreover, note that, if $\alpha$ is an integer, from (14) $\alpha \in\{0,1, . ., M-1\}$, and $\alpha \in E_{1}$ because $|\alpha-m|=0$ can appear only once.

$E_{2}$ Examination: The linear independence of the families $\sin \left(2 \pi \alpha_{m} \nu\right)$ and $\cos \left(2 \pi \alpha_{m} \nu\right)$, in the second sum in (17), yields: if $\cos (\beta) \sin (\beta)=\frac{1}{2} \sin (2 \beta) \neq 0$, i.e. $\beta \neq k \frac{\pi}{2}, k \in \mathbb{Z}$, the expression (17) holds only if $h_{m}+h_{m^{\prime}}=h_{m}-h_{m^{\prime}}=0$, i.e.:

$$
h_{m}=h_{m^{\prime}}=0 .
$$

This result imposes specific values to $\beta$ and coefficient pairing in order to obtain non-zero solutions, namely:

- $\beta=0[\pi]$, and in this case (17) holds if $h_{m}=h_{2 \alpha-m}, m \in E_{2}$, which corresponds to the symmetric case;

- $\beta=\frac{\pi}{2}+k \pi, k \in \mathbb{Z}$, and in this case (17) holds if $h_{m}=-h_{2 \alpha-m}, m \in E_{2}$, which corresponds to the antisymmetric case.

D. $\alpha$ Value

Let Min and Max be the indexes defined such as follows:

$$
\begin{aligned}
& \text { Min }=\underset{m}{\arg \min } h_{m} \neq 0, \\
& M a x=\arg \max _{m} h_{m} \neq 0 .
\end{aligned}
$$


The above definition of Min and Max is illustrated in Fig. 1, where $M=10$. In this example, it can be observed that $\operatorname{Min}=2$ and $\operatorname{Max}=8$. We now investigate the belonging of $\operatorname{Min}$ and Max to $E_{1}$ and $E_{2}$ in order to derive the $\alpha$ value:

- Case 1: Min and Max belong to different subsets. For instance, suppose that Min $\in E_{1}$ and $\operatorname{Max} \in E_{2}$. By examining $E_{1}$ in Section III-C, we know that $h_{M i n} \neq 0$, then $\alpha_{M i n}=0$, which yields $\alpha=$ Min and $\beta=k \pi, k \in \mathbb{Z}$. Since $\operatorname{Max} \in E_{2}, E_{2} \neq\{\emptyset\}$, which contains at least two indexes $m, m^{\prime}$ necessarily larger than Min. Hence $\alpha=\frac{m+m^{\prime}}{2}>$ Min, which is in contradiction with $\operatorname{Min}=\alpha$.

The same reasoning can be carried out when $\operatorname{Max} \in E_{1}$, which also leads to a contradiction. As a consequence, we deduce that both Min and $\operatorname{Max}$ belong to either $E_{1}$ or $E_{2}$, such as hereafter investigated.

- Case 2: Min, Max $\in E_{2}$ : we deduce from (14) that $h_{\text {Min }}=h_{2 \alpha-\operatorname{Min}}$ and $h_{M a x}=h_{2 \alpha-M a x}$, where $2 \alpha-\operatorname{Min}$ and $2 \alpha-\operatorname{Max}$ belong to $E_{2}$. Hence, we obtain Min $\leq 2 \alpha-\operatorname{Max}$ and $2 \alpha-\operatorname{Min} \leq \operatorname{Max}$ and therefore $\frac{\operatorname{Min}+\operatorname{Max}}{2} \leq \alpha \leq \frac{\operatorname{Min}+\operatorname{Max}}{2}$, i.e.:

$$
\alpha=\frac{\operatorname{Min}+\operatorname{Max}}{2} \text {. }
$$

Combined with those presented in Section III-C, the result in (21) highlights that the coefficients of the filter are (anti)symmetric to $\alpha$.

- Case 3: Min, Max $\in E_{1}$ : the unique trivial solution is $\operatorname{Min}=\operatorname{Max}=\alpha, \beta=k \pi, k \in \mathbb{Z}$. This result corresponds to the case where the filter is composed of a unique non-zero coefficient $h_{m}=h_{\alpha} \in \mathbb{R}$.

\section{E. Summary}

It has been shown that a constant group delay necessarily implies that:

- $\alpha=\frac{\operatorname{Min}+\operatorname{Max}}{2}$,

- $\beta \in\left\{k \pi, \frac{\pi}{2}+k \pi\right\}, k \in \mathbb{Z}$, and $\varepsilon_{\beta}=\left\{\begin{array}{ll}1, & \text { if } \beta=\frac{\pi}{2}+k \pi \\ -1, & \text { if } \beta=k \pi\end{array}\right.$.

- $h_{m}=\varepsilon_{\beta} h_{2 \alpha-m}$, which shows that the coefficients are (anti)symmetric to $\alpha$.

It must be emphasized that in practice, the extremal coefficients are non-zero values, i.e. Min $=0$ and $\operatorname{Max}=M-1$. Eventually, even if it means discarding null coefficients at the 
edges, we recognize the usual expression $\alpha=\frac{M-1}{2}$ such as defined in (10). Thus, we have shown that $(\mathcal{A}) \Rightarrow(\mathcal{B})$, which finally proves the equivalence $(\mathcal{A}) \Leftrightarrow(\mathcal{B})$.

\section{Conclusion}

In this paper, we proposed an original proof of the equivalence between the linear phase of FIR filters and the (anti)symmetry of the coefficients. As a prerequisite, the uniqueness of the group delay for any decomposition of the filter frequency response has been proved as well. The proof of equivalence has been carried out by considering a very general model, in which the taps of the filters are elements of a mathematical sequence. From this paradigm, we analyzed the independence of sin functions in order to deduce some properties of the FIR filters, namely the (anti)symmetry property of the coefficients, as well as the values of the linear phase parameters $\alpha$ and $\beta$. It is worth noting that the proposed proof remains valid if FIR filters with complex coefficients are considered. In that case, however, the coefficients are conjugate (anti)symmetric.

\section{REFERENCES}

[1] J. G. Proakis, D. G. Manolakis, Digital Signal Processing: Principles, Algorithms, and Applications, 4th Edition, Prentice Hall, 2007.

[2] L. R. Rabiner, J. H. McClellan, P. T. W., FIR Digital Filter Design Techniques Using Weighted Chebyshev Approximation, Proceedings of the IEEE 63 (4) (1975) 595 - 610.

[3] T. W. Parks, C. S. Burrus, Digital Filter Design (Topics in Digital Signal Processing), Wiley-Interscience, 1987.

[4] T. Saramäki, Handbook for Digital Signal Processing, John Wiley \& Sons, 1993, Ch. Chapter 4: Finite Impulse response design, pp. $155-277$.

[5] J. M. Pak, C. K. Ahn, P. Shi, Y. S. Shmaliy, L. M. T., Distributed Hybrid Particle/FIR Filtering for Mitigating NLOS Effects in TOA Based Localization Using Wireless Sensor Networks, IEEE Transactions on Industrial Electronics 64 (6) (2017) 5182 - 5191.

[6] C. K. Ahn, P. Shi, M. V. Basin, Deadbeat Dissipative FIR Filtering, IEEE Transaction on Circuits and Systems-I: Regular Papers 63 (8) (2016) 1210 - 1221.

[7] J. Bilmes, Lecture 15: Generalized Linear Phases and FIR Types, Tech. Rep. EE518, University of Washington, Seattle, Seattle (November 2001).

[8] P. S. R. Diniz, E. A. B. da Silva, S. L. Netto, Digital Signal Processing, System Analysis and Design, 2nd Edition, Cambridge University Press, 2002, Ch. 4: Digital Filters, pp. $222-276$.

[9] A. Hatcher, Algebraic Topology, 2001, Ch. Chapter 1: The Fundamental Group, pp. 21 - 96. 